${ }^{3}$ Stuart J, Barrett BA, Prangnell DR. Capillary blood collection in haematology. J Clin Pathol 1974;27:86974.

${ }^{4}$ Alder SM, Denton RL. The erythrocyte sedimentation rate in the newborn period. $J$ Paediatr 1975;86:942-8.

${ }^{5}$ Niejaldlik DC, Engelhardt C. An evaluation of the Guest method for determining erythrocyte sedimentation rate. Am J Clin Pathol 1977;68:766-8.

6 England NWJ, Warren DE, Rycroft S. Polystyrene ESR tubes. J Clin Pathol 1978;31:295.

${ }^{7}$ British Standards Institution.Westergren tube for measurement of erythrocyte sedimentation rate. British Standard 2554:1968.

${ }^{8}$ Moe PJ. Hemoglobin, hematocrit and red blood cell count in 'capillary' (skin prick) blood compared to venous blood in children. Acta Paediatr Scand 1970; 59:49-51.

Requests for reprints to: Dr GW Bird, Regional Blood Transfusion Centre, Vincent Drive, Birmingham B15 2SG.

\section{Saving tests by pooling sera-how great are the benefits?}

JY MORTIMER Epidemiological Research Laboratory, Central Public Health Laboratory, Colindale Avenue, London NW9 5HT, UK

When a large proportion of a population is negative for a serological factor, it may be possible to reduce the number of tests needed to identify positive individuals by first testing pooled samples and then re-testing individually the sera from any pool that gives a positive result.

If the proportion of negatives in the population is known, the pool size that will minimise the average number of tests needed to get a result for each of a set of sera can be readily established. Suppose sera from $N$ individuals, each with probability $P$ of being negative, are pooled in groups of size $k$, where $k$ is a factor of $N$. If all the individuals contributing to a pool are negative, only one test will be used. The probability of this happening is $\mathrm{P}^{k}$. If, however, the result from a pool is positive, an event occurring with probability $1-\mathrm{P}^{k}$, further $k$ tests will be needed for that pool. Thus the status of the $k$ individuals contributing to a pool will be determined by either 1 test, with probability $\mathrm{p}^{k}$ or $k+1$ tests, with probability $1-\mathrm{p}^{k}$. As $N / k$ is the number of pools, the expected number of tests needed to establish the status of the $N$ individuals is

$$
N / k \times\left(1 \times \mathrm{P}^{k}+(k+1) \times\left(1-\mathrm{P}^{k}\right)\right)
$$

which equals

$$
(N / k) \times\left(1+k\left(1-\mathrm{p}^{k}\right)\right)
$$

If $k$ is not a factor of $N$, there will be one or more sera, $r$ say, left after Int $(N / k)^{*}$ pools of size $k$ have been made. If these are treated as a separate pool of size $r$, the average number of tests needed to examine $N$ sera will be

$$
\begin{aligned}
\text { Int }(N / k) & \times\left(1+k\left(1-\mathrm{P}^{k}\right)\right) \\
& +1 \times\left(1+r\left(1-\mathrm{P}^{r}\right)\right)
\end{aligned}
$$

Alternatively, each of the remaining $r$ sera might be added to one of the pools of size $k$, which would make the average number of tests required

Int $(N / k)-r) \times\left(1+k\left(1-\mathrm{P}^{k}\right)\right)+$

$$
r \times\left(1+(k+1)\left(1-\mathrm{p}^{k+1}\right)\right.
$$

As the first practice is probably easier to manage in a laboratory, formula $B$ has been used to calculate the values for Table 1 , which shows the average number of tests needed to establish the status of 100 specimens with pool sizes from 2 to 11 for selected values of $P$, the proportion of negatives in the popu-

*Int $(N / k)$ is the whole number part of the quotient.

Received for publication 4 June 1980 
Table 1 Average number of tests needed to establish the status of 100 individuals for selected values of $P$

\begin{tabular}{|c|c|c|c|c|c|c|c|c|c|c|}
\hline \multirow{2}{*}{$\begin{array}{l}\text { Proportion of negatives } \\
\text { in population }(\mathrm{P})\end{array}$} & \multicolumn{10}{|c|}{ No. of sera included in each pool $(k)$} \\
\hline & 2 & 3 & 4 & 5 & 6 & 7 & 8 & 9 & 10 & 11 \\
\hline 0.999 & $50 \cdot 2$ & $34 \cdot 3$ & 25.4 & 20.5 & $17 \cdot 6$ & $15 \cdot 7$ & 13.8 & 12.9 & $11 \cdot 1$ & $11 \cdot 1$ \\
\hline 0.99 & 52.0 & 37.0 & 28.9 & 24.9 & $22 \cdot 8$ & $21 \cdot 7$ & $20 \cdot 6$ & $20 \cdot 6$ & $19 \cdot 6$ & $20 \cdot 4$ \\
\hline 0.95 & $59 \cdot 8$ & $48 \cdot 2$ & 43.5 & $42 \cdot 6$ & $43 \cdot 2$ & 44.8 & $46 \cdot 1$ & 48.7 & $50 \cdot 1$ & $52 \cdot 7$ \\
\hline 0.9 & $69 \cdot 0$ & 60.9 & 59.4 & $61 \cdot 0$ & $63 \cdot 4$ & 66.5 & $69 \cdot 1$ & $72 \cdot 7$ & $75 \cdot 1$ & 78.0 \\
\hline 0.8 & 86.0 & 82.5 & 84.0 & 87.2 & $90 \cdot 2$ & $93 \cdot 2$ & $95 \cdot 3$ & 97.9 & $99 \cdot 3$ & $100 \cdot 7$ \\
\hline $0 \cdot 6$ & 114.0 & 112.0 & 112.0 & 112.2 & 112.0 & 111.5 & 110.9 & $110 \cdot 4$ & $109 \cdot 4$ & $109 \cdot 0$ \\
\hline
\end{tabular}

Table 2 Ranges of pool sizes which perform relatively well at given values of $P$

\begin{tabular}{lcc}
\hline Proportion of negatives in population (P) & Range of values of $k$ (pool size) & $\begin{array}{l}\text { Minimum saving in average no. of tests } \\
\text { needed to find positive individuals }\end{array}$ \\
\hline 0.999 & $13-90$ & $90 \%$ \\
0.99 & $7-18$ & $78 \%$ \\
0.98 & $5-12$ & $70 \%$ \\
0.97 & $5-8$ & $65 \%$ \\
0.96 & $4-8$ & $59 \%$ \\
0.95 & $4-7$ & $55 \%$ \\
0.94 & $4-7$ & $50 \%$ \\
0.92 & $3-6$ & $44 \%$ \\
0.9 & $3-6$ & $36 \%$ \\
0.85 & $3-5$ & $24 \%$ \\
0.8 & $3-4$ & $16 \%$ \\
0.75 & $2-4$ & $6 \%$ \\
\hline
\end{tabular}

lation. This Table shows that if $99 \%$ of the population is negative, 10 is the most effective number of sera to include in each pool, and that the method would then, on average, reduce the number of tests used to $1 / 5$ when compared with testing each serum individually. If, on the other hand, only $90 \%$ of the population are negative, four would be the best size for each pool. The average number of tests used in these circumstances would be about $3 / 5$ of what would be required if the sera were not pooled. Pooling sera when the proportion of negatives in the population is too low will tend to increase the number of tests needed to above 100. From Table 1 it is also apparent that the precise pool size is not critical. For each proportion of negatives, $P$, there is a range of pool sizes about the optimum, all performing similarly.

Generally, of course, the proportion of negatives is not known. The purpose of the tests may be to establish it. It may be, too, that the nature of the test limits the number of sera that can be pooled, or makes some pool sizes more convenient than others. Testing and re-testing may, for instance, be greatly simplified by using specimens from one row of a rack to make each pool. A value of $k$ based on the rack design may, therefore, be desirable. For these reasons it is useful to know the range of values of $k$ that perform relatively well for given values of $\mathbf{P}$ (Table 2). A pool size may be chosen from this table using any available knowledge of the proportion of negatives in the population. The expected number of tests required for a given $N$, selected $k$, and estimated $\mathbf{P}$ may be calculated using formula $\mathrm{A}, \mathrm{B}$, or $\mathrm{C}$, as appropriate.

The procedure should not be used with a test that might fail to detect a weakly positive serum in an otherwise negative pool. It will also be inappropriate if a negative serum can mask a positive by, for instance, an antigen-antibody reaction. If the method is applicable, its usefulness will be largely determined by the proportion of negatives in the population concerned. If positives are rarer than 1 in 100 , it will often be beneficial to pool sera as a pool size as small as two will almost halve the number of tests required, and larger pools will produce much greater savings. When positives are more common, up to 1 in 10, say, the value of pooling will depend on the nature of the test. It will be worthwhile only when its cost and complexity outweigh the extra labour involved in making the pools and re-testing the constituents of positive ones individually. For serological factors expected to have a higher incidence than 1 in 10 it is unlikely that pooling will produce a benefit. Indeed, an over-estimate of the proportion of negatives involved may result in the use of more tests than would have been needed to test sera individually in the first place.

Requests for reprints to: Mrs JY Mortimer, Epidemiological Research Laboratory, Central Public Health Laboratory, Colindale Avenue, London NW9 5HT. 